\title{
High level perinuclear antineutrophil cytoplasmic antibody (pANCA) in ulcerative colitis patients before colectomy predicts the development of chronic pouchitis after ileal pouch-anal anastomosis
}

P R Fleshner, E A Vasiliauskas, L Y Kam, N E Fleshner, J Gaiennie, M T Abreu-Martin, S R Targan

\begin{abstract}
Background-The reported cumulative risk of developing pouchitis in ulcerative colitis (UC) patients undergoing ileal pouch-anal anastomosis (IPAA) approaches $50 \%$ after 10 years. To date, no preoperative serological predictor of pouchitis has been found.

Aims-To assess whether preoperative perinuclear antineutrophil cytoplasmic antibody (pANCA) expression was associated with acute and/or chronic pouchitis after IPAA.

Methods-Patients were prospectively assessed for the development of clinically and endoscopically proved pouchitis. Serum obtained at the time of colectomy in $95 \mathrm{UC}$ patients undergoing IPAA was analysed for pANCA by ELISA and indirect immunofluorescence. pANCA+ patients were stratified into high level $(>100$ ELISA units $(E U) / m l)(n=9)$, moderate level (40-100 EU/ml) (n=32), and low level $(<40 \mathrm{EU} / \mathrm{ml})(\mathrm{n}=19)$ subgroups.
\end{abstract}

Results-Sixty of the 95 patients (63\%) expressed pANCA. After a median follow up of 32 months (range 1-89), 32 patients $(34 \%)$ developed either acute $(n=14)$ or chronic $(n=18)$ pouchitis. Pouchitis was seen in $42 \%$ of pANCA+ patients compared with $20 \%$ of pANCA- patients $(p=0.09)$. There was no significant difference in the incidence of acute pouchitis between the three pANCA+ patient subgroups. The cumulative risk of developing chronic pouchitis among patients with high level pANCA (56\%) before colectomy was significantly higher than in patients with medium level (22\%), low level $(16 \%)$, and those who were pANCA- $(20 \%)(p=0.005)$. Multivariate analysis revealed that the sole parameter significantly associated with the development of chronic pouchitis after IPAA was the presence of high level pANCA before colectomy $(p=0.005)$. Conclusion-High level pANCA before colectomy is significantly associated with the development of chronic pouchitis after IPAA.

(Gut 2001;49:671-677)

Keywords: pouchitis; perinuclear antineutrophil cytoplasmic antibody; inflammatory bowel disease; ulcerative colitis; ileal pouch-anal anastomosis

Abdominal colectomy with ileal pouch-anal anastomosis (IPAA) has become the standard operative approach for patients with ulcerative colitis (UC) requiring colectomy for dysplasia, cancer, or medically unresponsive disease. This procedure removes all diseased mucosa while preserving continence and transanal defecation and is associated with excellent functional results and high patient satisfaction..$^{1-3}$

The most frequent long term complication after IPAA for UC is a non-specific inflammation of the ileal pouch commonly known as pouchitis. $^{2-4}$ The presence of extraintestinal manifestations (EIMs) of UC before colectomy, especially primary sclerosing cholangitis (PSC), has been associated with the development of pouchitis. ${ }^{4}$ However, the positive predictive value of EIMs for pouchitis is low, and only about $25 \%$ of all UC patients ever have EIMs. ${ }^{4}$ Although the positive predictive value of PSC for pouchitis is higher, fewer than $5 \%$ of all UC patients ever develop PSC. ${ }^{5}$ A more clinically useful predictor of pouchitis occurrence is clearly needed.

Serum antineutrophil cytoplasmic antibodies with perinuclear staining (pANCA) have been detected in the majority of UC patients before and after IPAA. ${ }^{6-9}$ Recent studies have revealed that expression of this marker antibody reflects an immune response to the antigen products of enteric bacteria. ${ }^{10}{ }^{11}$ Seibold et al demonstrated that pANCA cross reacts with mouse caecal bacterial antigens. ${ }^{10}$ In addition, Cohavy et al discovered that pANCA binds to the Escherichia coli derived outer membrane porin OmpC. ${ }^{11}$ Although the search for an infectious agent that causes pouchitis has not been successful, there is ample evidence to suggest that enteric bacteria and their products are involved in the pathogenesis of this disease. In contrast with patients with adenomatous polyposis coli, who rarely develop pouchitis after IPAA, UC patients are susceptible to the development of mucosal inflammation after colonisation of the ileal pouch by intestinal

Abbreviations used in this paper: UC, ulcerative colitis; PSC, primary sclerosing cholangitis; pANCA, perinuclear antineutrophil cytoplasmic antibody; IPAA, ileal pouch-anal anastomosis; EIM, extraintestinal manifestations; EU, ELISA unit. 
bacteria. $^{2}{ }^{12-15}$ Additionally, treatment of pouchitis with antibiotics is almost universally successful. ${ }^{16}$

These observations together suggest that pANCA may represent a serological marker for pouchitis development after IPAA. Prior studies examining the correlation between pANCA expression and pouchitis have been contradictory, with three studies showing a positive association ${ }^{17-19}$ and some studies demonstrating no significant difference in pANCA expression between patients with or without pouchitis. ${ }^{20-22}$ All of these studies were retrospective in design, many measured pANCA at the time of pouchitis diagnosis and not before surgery, and some examined patients with treatment resistant pouchitis and did not evaluate the more common acute or chronic treatment responsive pouchitis. Additionally, the association between pANCA expression and acute pouchitis has not been addressed. This study was specifically designed to address these methodological problems.

The goal of this prospective study was to assess whether preoperative pANCA expression was associated with acute and/or chronic pouchitis after IPAA. Additionally, as binding of pANCA to bacterial antigens has only been identified in patients with high level antibody expression, ${ }^{11}$ we also assessed whether preoperative levels had any association with the incidence of acute and/or chronic pouchitis after IPAA. The results demonstrate the significance of taking into account the magnitude of the host immune response, in that high level pANCA expression before colectomy was associated with an increased incidence of chronic pouchitis after IPAA.

\section{Materials and methods}

STUDY POPULATION

As part of a prospective study to correlate serological and genetic markers with clinical phenotypes in inflammatory bowel disease (IBD), a cohort of 95 UC patients requiring colectomy for medically unresponsive disease or dysplasia was studied. All patients operated on at Cedars-Sinai Medical Center by one surgeon (PRF) over the six year period ending March 2000 were eligible for the study. Complete mucosectomy was performed in all patients. In addition, all patients had a temporary diverting ileostomy constructed at the time of pouch creation. All patients had clinical, radiological, and histological features diagnostic of UC. Clinically, perianal disease was absent, and endoscopic features included continuous macroscopic disease extending varying distances proximally from the dentate line. Radiological evaluation revealed the distinct absence of either a colonic stricture or small bowel disease. Histological patterns of continuous microscopic inflammation and absence of non-caseating granulomas were mandatory to rule out Crohn's disease. Additionally, these features were also identified after review of intraoperative findings and pathological evaluation of the resected specimen. Patients with indeterminate colitis, those in whom the ileostomy was not reversed, and those who did not consent to have blood drawn for research purposes were not included in the study population. Patients with recognised mechanical complications of surgery such as an anal stricture or anastomotic dehiscence were also specifically excluded.

ASSESSMENT OF CLINICAL CHARACTERISTICS Detailed patient profiles were prospectively generated by one of the investigators (PRF) who was blinded to individual patients' ANCA status. Clinical information was collected by chart review and patient interview. Patients were seen for follow up examinations every three months for the first year after stoma closure and yearly afterwards. Clinical characteristics examined included patient age at surgery, age at diagnosis of UC, disease duration, sex, family history of IBD, anatomical location of UC, EIMs of IBD, the presence or absence of backwash ileitis, indications for surgery, and the development of and time to pouchitis. Anatomical location of disease was grouped into the categories of pancolitis, left sided colitis, and proctitis. EIMs included PSC and skin lesions (pyoderma gangrenosum, erythema nodosum), bone/joint disease (arthritis, ankylosing spondylitis, sacroileitis), or eye disease (uveitis, episcleritis) considered by the investigators and the patients' physicians to be manifestations of IBD. Backwash ileitis was defined by the presence of macroscopic or histological evidence of inflammation restricted to the distal $3 \mathrm{~cm}$ of the extreme terminal ileum not thought to be related to Crohn's disease. Indications for surgery included medically unresponsive disease, dysplasia, or cancer.

DIAGNOSIS OF POUCHITIS

Pouchitis was defined as a clinical syndrome characterised by the onset of increased stool frequency often with bloody diarrhoea, pelvic discomfort, urgency, malaise, and fever. The diagnosis of pouchitis was confirmed in all cases by flexible endoscopy. Endoscopic findings in cases of pouchitis included diffuse mucosal inflammation, typically involving the entire pouch, characterised by exudate, ulceration, and erythema, and sparing of the proximal ileum. Acute pouchitis was defined as being antibiotic responsive flares occurring at least four months apart during which time the patient was completely asymptomatic and had returned to his/her usual bowel pattern. Chronic pouchitis required continuous antibiotic treatment for symptom relief and also included those patients who were refractory to antibiotic treatment. Stool cultures were obtained when conventional antibiotic therapy was unsuccessful or in patients with chronic pouchitis. Histological evaluation of the pouch was not routinely performed. For patients with persistent symptoms, studies were done to exclude mechanical complications of surgery such as an anal stricture or partial small bowel obstruction. Time to diagnosis of pouchitis was defined as the time period from ileostomy closure. 
SERUM ANCA DETERMINATION AND ANCA SUBTYPE CHARACTERISATION

Study patient sera were drawn immediately before colectomy, coded, and stored for future analysis. Serum analysis for ANCA expression was performed in a blinded fashion by Prometheus Laboratories (San Diego, California, USA). ANCA presence was determined by fixed neutrophil ELISA, as previously described. ${ }^{6}$ Polystyrene microtitre plates were coated with $2.5 \times 10^{5}$ normal human donor whole peripheral blood neutrophils per well, which were then fixed with $100 \%$ methanol. Cells were incubated with bovine serum albumin $(0.25 \%)$ in phosphate buffered saline to block non-specific antibody binding. Next, control and coded sera were added at a 1:100 dilution. Following incubation and washing, goat $\mathrm{F}\left(\mathrm{ab}^{\prime}\right)_{2}$ antihuman immunoglobulin $\mathrm{G}(\gamma$ chain specific) antibody (Jackson Immunoresearch Labs, Inc., West Grove, Pennsylvania, USA) conjugated to alkaline phosphatase was added at a 1:1000 dilution to label neutrophil bound antibody. Substrate solution containing $p$-nitrophenol phosphate was then added. Colour development was allowed to proceed until absorbence at $405 \mathrm{~nm}$, with a reference wavelength of $650 \mathrm{~nm}$ on a Molecular Devices (Menlo Park, California, USA) E-Max microtitre plate reader, in the positive control wells was $0.8-1.0$ optical density units greater than that in the blank wells. Levels were determined relative to a Prometheus laboratory standard consisting of pooled sera obtained from well characterised pANCA+ UC patients. Results were expressed as ELISA units $(\mathrm{EU} / \mathrm{ml})$. Sera with circulating antineutrophil cytoplasmic IgG antibody exceeding the normal reference range value were termed "ANCA+." Values that were negative relative to the panel of pooled pANCA+ control sera were termed "ANCA-."

ANCA+ sera were further subtyped via indirect immunofluorescence staining to determine the ANCA neutrophil binding pattern. Glass slides containing approximately $10^{5}$ normal human donor whole peripheral blood neutrophils per slide were prepared by cytocentrifugation (Shandon Cytospin, Cheshire, UK). Cells were fixed in $100 \%$ methanol, air dried, and stored at $-20^{\circ} \mathrm{C}$. The coded patient sera were diluted $(1: 20)$ and then layered over the fixed neutrophils, incubated at $37^{\circ} \mathrm{C}$, and then washed. Fluorescein labelled $\mathrm{F}\left(\mathrm{ab}^{\prime}\right)_{2} \gamma$ chain specific goat antihuman antibody was added and incubated, and then washed, as previously described. ${ }^{6}$ The sections were examined by fluorescence microscopy using an epifluorescence equipped Nikon microscope (Garden City, New Jersey, USA). Sera exhibiting the characteristic perinuclear highlighting which then lost this characteristic staining pattern when first treated with DNase were termed "pANCA+". ${ }^{23}$

STRATIFICATION OF pANCA+ PATIENTS BASED ON ANTIBODY LEVEL

A recent study by Vasiliauskas et al demonstrated that the level of serum immune markers, not only their absence or presence, allows for stratification of IBD patients into more homogenous immunologically distinct subgroups with common subclinical and clinical features. ${ }^{24}$ Cohavy et al, in examining the association between pANCA levels and binding to the Escherichia coli outer membrane porin OmpC, found that only sera with high pANCA levels (titres $>1 / 1000$, which is equivalent to $100 \mathrm{EU} / \mathrm{ml}$ ) had significant bacterial antigen binding. ${ }^{11}$ These data suggest that high level pANCA expression may identify a subset of patients with UC who may have a distinct type of mucosal inflammation representing an exquisite sensitivity to the presence of bacterial antigens. These biological models stimulated our analysis of patients based on quantitative pANCA antibody expression. pANCA+ patients were thus stratified into three groups based on antibody level: high level pANCA patients had levels $>100 \mathrm{EU} / \mathrm{ml}$; medium level, 40-100 EU/ml; and low level, <40 EU/ml.

\section{STATISTICAL ANALYSIS}

All data were entered into a standardised database computer program (Microsoft, Seattle, Washington, USA). Statistical analyses were performed with the SPSS (SPSS, Chicago, Illinois, USA) statistical package for Windows. We first logarithmically transformed ANCA levels (including those below the reference range) to obtain a more ready normal distribution before correlation was computed and statistical tests were performed. Correlations between normalised variables were evaluated by Pearson correlation coefficients and corresponding $\mathrm{p}$ values were calculated. To evaluate the relationship between serum pANCA and baseline clinical features, linear regression analysis was performed by specifying antibody levels as dependent variables and clinical characteristics as independent variables, using a stepwise procedure. For continuous covariates, means were compared with the use of the Student's $t$ test. Categorical variables were compared with the use of the $\chi^{2}$ method or Fisher's exact test (if expected cell counts were less than 5). Chronic pouchitis free survival was then plotted using the Kaplan-Meier method for the various tested covariates. The log rank test was used to compare these survival curves for the various substrata. Finally, multivariate analyses of chronic pouchitis free survival were performed using the proportional hazards model according to Cox. All hypothesis testing was two sided with $p$ values less than 0.05 considered statistically significant. Due to the hypothesis generating nature of this study, a Bonferroni correction was not used.

\section{Results}

PATIENT DEMOGRAPHICS AND CLINICAL

CHARACTERISTICS

Sixty one of the 95 study patients $(64 \%)$ were male (table 1). Of the 74 patients with medically unresponsive disease, 19 (26\%) were steroid dependent and $55(74 \%)$ were refractory to other immunomodulatory therapies, including 6-mercaptopurine $(n=51)$ and/or cyclosporin $(n=37)$. Median follow up time 
Table 1 Patient characteristics of the study population

\begin{tabular}{ll}
\hline Sex $(M / F)$ & $61 / 34$ \\
Age (y) (median (range)) & $40(11-78)$ \\
Disease duration (months) (median (range)) & $72(4-456)$ \\
Disease extent & 72 \\
$\quad$ Pancolitis & 20 \\
Left sided & 3 \\
$\quad$ Proctitis & 8 \\
Extraintestinal disease & 3 \\
$\quad$ Arthritis & 3 \\
PSC & 74 \\
Indication for IPAA & 12 \\
$\quad$ Medically unresponsive & 9 \\
Cancer & 8 \\
Dysplasia & \\
Backwash ileitis &
\end{tabular}

PSC, primary sclerosing cholangitis; IPAA, ileal pouch-anal anastomosis.

after ileostomy closure was 32 months (range 1-89). No patient was lost to follow up.

POUCHITIS

Thirty two patients developed pouchitis, representing an overall incidence of $34 \%$. Acute pouchitis was seen in 14 patients $(15 \%)$ and chronic pouchitis in $18(19 \%)$. Median time to diagnosis of pouchitis in the study group was five months (range 1-63). There was no difference in median time to pouchitis diagnosis between patients with acute (five months) or chronic (six months) pouchitis. In addition, patient age, sex, disease extent, extraintestinal disease, indication for surgery, use of immunomodulatory drugs, and the presence of backwash ileitis were not significantly different between the two pouchitis groups.

RELATIONSHIP BETWEEN PANCA EXPRESSION AND POUCHITIS

Serum ANCA was detected in 82 patients $(86 \%)$ in samples taken before colectomy. Sixty patients $(63 \%)$ had a perinuclear staining pattern which disappeared with DNAse treatment (pANCA+). There was no significant difference in patient demographics or disease characteristics between the DNAse sensitive pANCA+ and pANCA- patient groups.

The association between pouchitis and preoperative pANCA status is shown in fig 1 . There was no significant difference in the overall incidence of pouchitis between the pANCA+ and pANCA- patient groups $(p=0.09)$. The incidence of acute pouchitis was also not significantly different between these two patient groups. Although the incidence of chronic pouchitis was higher in the pANCA+ patient group $(25 \%)$ than in the pANCApatient group $(9 \%)$, this difference was not statistically significant $(\mathrm{p}=0.09)$.

RELATIONSHIP BETWEEN PANCA ANTIBODY LEVEL AND POUCHITIS

The magnitude of preoperative pANCA expression was evaluated quantitatively by the ELISA level of host marker antibody production before colectomy. The median preoperative antibody level in the pANCA+ patient group was $56 \mathrm{EU} / \mathrm{ml}$. However, there was a wide range of preoperative antibody levels noted in this patient group, ranging from 10 to $170 \mathrm{EU} / \mathrm{ml}$ (fig 2). There were nine patients

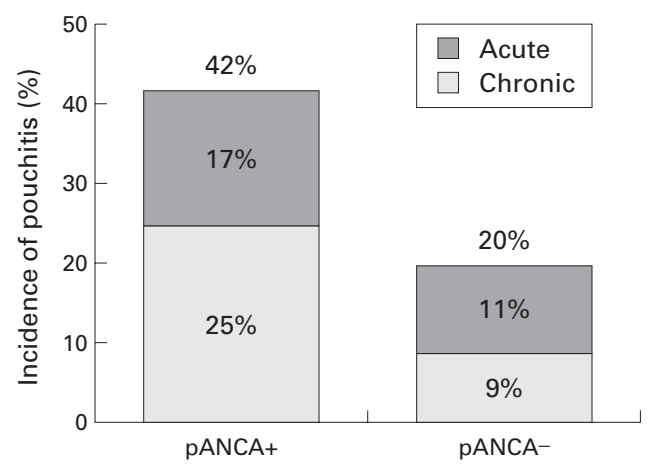

Figure 1 Incidence of pouchitis in the perinuclear antineutrophil cytoplasmic antibody positive ( $p A N C A+)$ and negative ( $p A N C A-)$ patient groups. There was no significant difference in the incidence of acute $(p=0.7)$ or chronic $(p=0.09)$ pouchitis between the $p A N C A+$ and pANCA-patient groups

$(15 \%)$ in the high level group, 32 patients in the medium level group (53\%), and 19 patients $(32 \%)$ in the low level group (see methods). Patient characteristics were not significantly different between the three different pANCA+ groups (table 2). Multiple regression analysis using quantitative antibody level as the dependent variable also confirmed no significant relationship between pANCA level and preoperative patient clinical features (table 3 ).

The incidence of pouchitis noted in the three different pANCA+ patient groups is shown in fig 3. The $78 \%$ incidence of pouchitis in the high level pANCA+ patient group was higher than the $41 \%$ incidence of pouchitis noted in the medium level pANCA+ patient group and significantly higher than the $22 \%$ incidence in the low level pANCA+ patient group $(\mathrm{p}=$ 0.03 ). Interestingly, the $22 \%$ incidence of pouchitis noted in the low level pANCA+ patient group was similar to the pANCA- patient group (20\%). Although there was a significantly higher incidence of pouchitis in the high level pANCA+ patient group, this difference was accounted for solely on the basis of chronic pouchitis $(p=0.03)$. There was no significant difference in the incidence of acute pouchitis between the three different pANCA+ patient groups.

Kaplan-Meier analysis was undertaken to assess the risk of chronic pouchitis among patients with different pANCA levels before colectomy (fig 4). The risk of developing

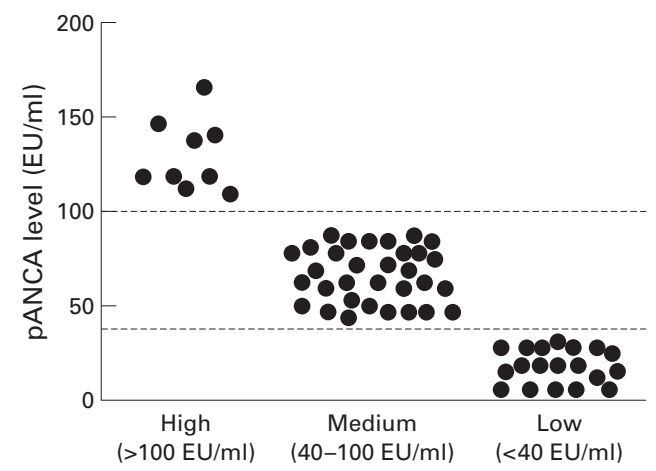

Figure 2 Preoperative antibody titres (EU/ml) in the high level, medium level, and low level perinuclear antineutrophil cytoplasmic antibody positive ( $p A N C A+)$ patient groups. 
Table 2 Patient characteristics in the three different perinuclear antineutrophil cytoplasmic antibody positive $(p A N C A+)$ groups

\begin{tabular}{llll}
\hline & $\begin{array}{l}\text { High level } \\
(n=9)\end{array}$ & $\begin{array}{l}\text { Medium level } \\
(n=32)\end{array}$ & $\begin{array}{l}\text { Low level } \\
(n=19)\end{array}$ \\
\hline Sex (M/F) & $6 / 3$ & $17 / 15$ & $16 / 3$ \\
Age (y) (median (range)) & $34(16-63)$ & $38(20-78)$ & $41(14-67)$ \\
Disease duration (months) (median (range)) & $40(17-180)$ & $96(6-408)$ & $48(4-360)$ \\
Disease extent (n) & 6 & 25 & 17 \\
$\quad$ Pancolitis & 3 & 5 & 1 \\
$\quad$ Left sided & 0 & 2 & 1 \\
$\quad$ Proctitis & 8 & 25 & 17 \\
$\quad$ Indication for IPAA (n) & 1 & 7 & 2 \\
$\quad$ Medically unresponsive & 2 & 4 & 1 \\
$\quad$ Bancer/dysplasia & 0 & 2 & 4 \\
Extraintestinal disease (n) & 1 & 4 & \\
$\quad$ PSC & 1 & & \\
$\quad$ Other & & 25 & \\
\hline
\end{tabular}

IPAA, ileal pouch-anal anastomosis; PSC primary sclerosing cholangitis.

There were no significant differences between the three patient groups.

chronic pouchitis after IPAA among patients with high level pANCA before colectomy was significantly higher than in patients with medium level pANCA, low level pANCA, and patients who were $\mathrm{pANCA}-$ (overall $\mathrm{p}=0.005$ ).

Multivariate analysis revealed that the sole demographic or clinical characteristic significantly associated with the development of chronic pouchitis after IPAA was high level pANCA expression before colectomy $(p=0.005)$. The relative risk of developing chronic pouchitis in this patient group was more than eightfold higher (hazard ratio 8.47;

Table 3 Linear multiple regression analysis using perinuclear antineutrophil cytoplasmic antibody ( $p A N C A$ ) levels as the dependent variable

\begin{tabular}{lrll}
\hline Clinical feature & \multicolumn{1}{l}{$\beta$} & $r^{2}$ & $p$ Value \\
\hline Sex & 0.039 & 0.004 & 0.55 \\
Age at surgery & -0.124 & 0.029 & 0.72 \\
Duration of disease & -0.535 & 0.025 & 0.81 \\
Family history of IBD & 0.138 & 0.017 & 0.21 \\
Disease extent & 0.075 & 0.003 & 0.50 \\
Extraintestinal disease & 0.019 & 0.001 & 0.85 \\
Backwash ileitis & -0.215 & 0.043 & 0.06 \\
Indication for surgery & -0.084 & 0.011 & 0.47
\end{tabular}

$\beta$, regression coefficient; $r^{2}$, proportion of the total variation of a dependent variable that can be explained by the variation of each independent variable; IBD, inflammatory bowel disease.

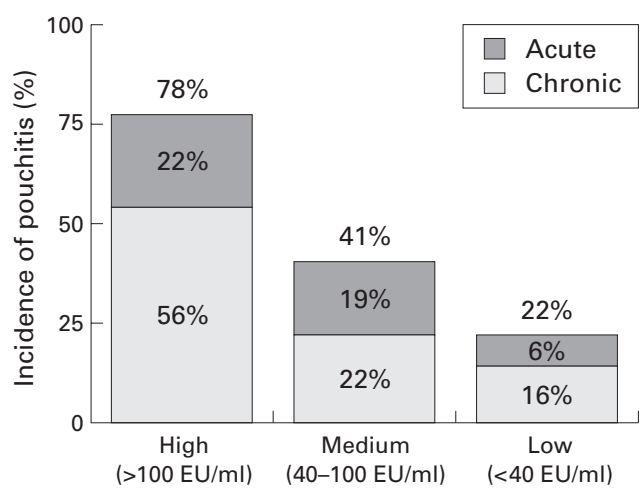

Figure 3 Incidence of acute and chronic pouchitis in the high level, medium level, and low level perinuclear antineutrophil cytoplasmic antibody positive ( $p A N C A+)$ patient groups. Although there was a significantly higher overall incidence of pouchitis in the high level $p A N C A+$ patient group, this difference was accounted for solely on the basis of chronic pouchitis $(p=0.03)$. There was no significant difference in the incidence of acute pouchitis $(p=0.21)$ between the three different $p A N C A+$ patient groups

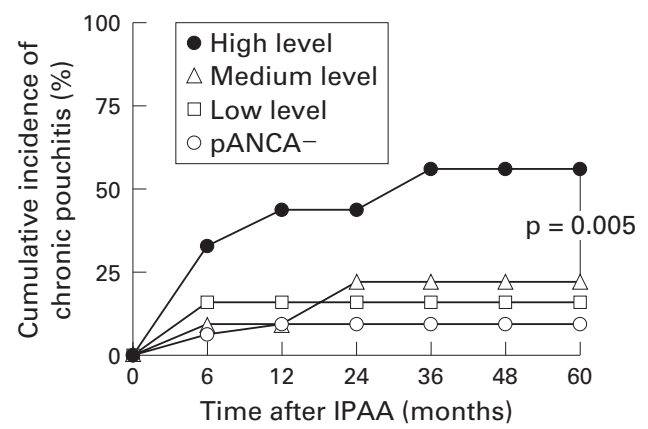

Figure 4 Cumulative incidence of chronic pouchitis in the high level, medium level, and low level perinuclear antineutrophil cytoplasmic antibody positive ( $p A N C A+)$ patient groups and the $p A N C A-$ patient group. The risk of developing chronic pouchitis after IPAA among patients with high level $p A N C A$ before colectomy was significantly higher than in patients with medium level $p A N C A$, low level $p A N C A$, and patients who were $p A N C A-$ (overall $p=0.005$ ).

Table 4 Multivariate analysis for the development of chronic pouchitis

\begin{tabular}{llll}
\hline Covariate & HR & $95 \%$ CI & p Value \\
\hline Sex & & & \\
$\quad$ Male & 1 & N/A & 0.06 \\
$\quad$ Female & 2.95 & $1.0-8.47$ & \\
Age at surgery & 0.98 & $0.94-1.02$ & 0.26 \\
$\begin{array}{l}\text { Family history } \\
\quad \text { No }\end{array}$ & 1 & N/A & \\
$\quad$ Yes & 1.89 & $0.63-5.55$ & 0.26 \\
Disease extent & & & \\
$\quad$ Left sided & 1 & N/A & \\
$\quad$ Pancolitis & 1.96 & $0.60-6.06$ & 0.26 \\
Extraintestinal disease & & & \\
$\quad$ No & 1 & N/A & \\
$\quad$ Yes & 2.33 & $0.71-7.69$ & 0.16 \\
High level pANCA & & & \\
$\quad$ No & 1 & N/A & \\
$\quad$ Yes & 8.47 & $1.67-16.95$ & 0.005 \\
\hline
\end{tabular}

HR, hazard ratio; CI, confidence interval; pANCA, perinuclear antineutrophil cytoplasmic antibody positive; N/A, not applicable.

95\% confidence interval 1.67-16.95) compared with patients who did not express high level pANCA before colectomy (table 4).

\section{Discussion}

Many centres around the world have shown pANCA to be present in the sera of the majority of UC patients before colectomy, with the incidence in most series ranging from $60 \%$ to $80 \% .^{92-27}$ Clinically, pANCA expression in $\mathrm{UC}$ is associated with distinct clinical phenotypes, including treatment resistant left sided disease, ${ }^{28}$ aggressive disease course, ${ }^{29}{ }^{30}$ and the need for surgical intervention early in the disease course. ${ }^{31}$ These studies suggest that serum pANCA production in UC reflects a distinct type of mucosal inflammation. The present study demonstrates that high level pANCA expression before colectomy was associated with the development of chronic pouchitis after IPAA. Although prior studies of pANCA in UC have focused on its absence or presence at random points after IPAA, our study is the first to observe that the magnitude of pANCA expression prior to colectomy, as measured by immune marker level, was associated with a specific clinical phenotype in UC patients. The findings suggest that expression 
of this immune marker may reflect different inflammatory mechanisms that influence disease expression (that is, pouchitis development).

Pouchitis is the most frequent complication of transanal continent reservoirs in patients undergoing IPAA. ${ }^{2-4}$ "Pouch ileitis" was first described by Kock et al in patients with a continent ileostomy and was characterised by bacteriological and histological changes within the pouch. ${ }^{32}$ Today, pouchitis is understood to be an acute or chronic inflammatory condition of unknown aetiology that affects both transanal continent reservoirs and continent ileostomy pouches. The frequency of pouchitis in patients undergoing IPAA varies between 15\% and $51 \% .^{1-5} 12-1533-38$ The wide variability of reporting of pouchitis frequency in the literature can be accounted for primarily because of a lack of accepted diagnostic criteria. Some authors define pouchitis on clinical grounds alone, ${ }^{2-5} 83335$ others require endoscopic confirmation $^{1} 1518343637$ while still other groups require that specific histopathological criteria be met. ${ }^{12-14} 1719223839$ Using clinical, endoscopic, and specific histopathological criteria, Sandborn et al have proposed an index to assess pouchitis activity. ${ }^{40}$ In our opinion, a combination of symptoms and endoscopic evidence of mucosal inflammation obviates the need for a biopsy and histopathological evaluation in most patients. Our overall incidence of pouchitis of $34 \%$ is in the range reported by other investigators who use similar diagnostic criteria. $^{11518343637}$

Prior studies examining the correlation between pANCA expression and pouchitis have been contradictory. ${ }^{17-22}$ Three studies found no association between the incidence of pouchitis and pANCA expression. ${ }^{17-19}$ Aisenberg et al found that the frequency of pANCA in IPAA patients with "refractory" pouchitis was not significantly different than that in IPAA patients without pouchitis. However, determination of pANCA was undertaken at the time of pouchitis diagnosis, not before surgery, and levels were not considered. Furthermore, their study examined patients with treatment resistant pouchitis and did not evaluate the more common chronic treatment responsive pouchitis. ${ }^{17}$ Although two other studies found no significant difference in pANCA expression between patients with or without pouchitis, ${ }^{18}{ }^{19}$ their retrospective study design and measurement of pANCA at variable times after colectomy do not firmly refute the reliability of using pANCA as a preoperative marker to identify those patients who may develop pouchitis. On the other hand, Sandborn et al from the Mayo Clinic measured pANCA in a cohort of adult UC patients who had already undergone IPAA and found that the frequency of pANCA in patients with chronic pouchitis was $100 \%$ compared with only $50 \%$ in patients without pouchitis. ${ }^{21}$ Two other studies have corroborated these results. $^{20}{ }^{22}$ These three studies taken together suggest that pANCA may predict the development of pouchitis after IPAA; however, the retrospective nature of these studies could not answer whether determination of pANCA level before colectomy could predict pouchitis after surgery. Additionally, they did not fully examine the association between pANCA expression before colectomy and acute pouchitis.

This study was designed to address the methodological problems associated with prior studies examining the influence of pANCA expression on pouchitis development. A true assessment of the potential value of pANCA as a serological marker for pouchitis development was assured by drawing patient sera immediately before colectomy. All patients were followed prospectively after IPAA for the development of pouchitis using strict clinical and endoscopic criteria by a single investigator blinded to the patients' pANCA status. In addition, pANCA determination was performed without knowledge of the patients' clinical status. Our study design also allowed for the assessment of both acute and chronic pouchitis. Finally, host antibody expression and level were measured using a three step method, an approach which has recently been shown to be the most sensitive pANCA assay in a population based cohort. ${ }^{41}$

The incidence of either acute or chronic pouchitis in the pANCA+ patient group was not significantly higher than that in the pANCA- patient group. Accordingly, preoperative pANCA expression alone does not appear to predict pouchitis after colectomy and IPAA, corroborating the results of other studies. ${ }^{17-19}$ However, we uncovered a number of interesting observations in examining the incidence of pouchitis in patients with different pANCA levels. We felt this subgroup analysis was needed based on evidence that: (1) pouchitis is a bacteria mediated immunological disease $^{212-16}$ and (2) the results of a recent study which revealed significant binding only with sera from UC patients with high level pANCA $(>100 \mathrm{EU} / \mathrm{ml})$ and a bacterial wall antigen (OmpC) located on the outer membrane wall of Escherichia coli. ${ }^{11}$ The overall incidence of pouchitis was significantly influenced by the presence of high level pANCA before colectomy. This correlation was due primarily to the effect of high level pANCA on the incidence of chronic pouchitis. The pathogenetic mechanisms accounting for this association remain unknown. It is possible that patients with high level pANCA are predisposed to develop a vigorous immune response to bacterial products. Perhaps further characterisation of the role that OmpC or other bacterial antigens play in the inflammatory cascade might provide insight into these issues and delineate the pathogenesis of pouchitis and even UC itself.

Clinically, this study suggests that patients with high level pANCA before colectomy should be counselled on their higher risk of developing chronic pouchitis after surgery and the potential need for long term antibiotic treatment. However, as most patients with chronic pouchitis are clinically improved with antibiotic therapy, ${ }^{16}$ patients would probably still choose the risks of long term antibiotic treatment in lieu of a permanent ileostomy. Additionally, as high level pANCA expression 
before colectomy for UC is an important predictor of chronic pouchitis after IPAA, it would be interesting to investigate whether prophylactic antibiotic therapy in UC patients after IPAA having high level pANCA expression before colectomy could prevent or delay pouchitis development.

In conclusion, high level pANCA expression before colectomy predicts chronic but not acute pouchitis after IPAA. Patients with high level pANCA may represent a distinct subgroup of UC patients that manifest different clinical behaviour after IPAA.

The authors would like to acknowledge Loren Karp for editoria assistance. Supported by USPHS grant PO 1 DK46763 and the Feintech Family Chair in Inflammatory Bowel Disease.

1 Marcello PW, Roberts PL, Schoetz DJ, et al. Long-term results of the ileoanal pouch procedure. Arch Surg 1993;128:500-4

2 Fazio VW, Ziv Y, Church JM, et al. Ileal pouch-anal anastomoses complications and function in 1005 patients. Ann Surg 1995;222:120-7.

3 Meagher AP, Farouk R, Dozois RR, et al. J ileal pouch-anal anastomosis for chronic ulcerative colitis: complications and long-term outcome in 1310 patients. Br f Surg 1998;85:800-3

4 Lohmuller JL, Pemberton JH, Dozois RR, et al. Pouchitis and extraintestinal manifestations of inflammatory bowel
disease after ileal pouch-anal anastomosis. Ann Surg 1990; 211:622-7.

5 Penna C, Dozois R, Tremaine W, et al. Pouchitis after ileal pouch-anal anastomosis for ulcerative colitis occurs with pouch-anal anastomosis for ulcerative colitis occurs with
increased frequency in patients with associated primary increased frequency in patients with associa

6 Saxon A, Shanahan F, Landers C, et al. A distinct subset of antineutrophil cytoplasmic antibodies is associated with inflammatory bowel disease. F Allergy Clin Immuno 1990;86:202-10.

7 Oudkerk-Pool M, Ellerbroek PM, Ridwan BU, et al. Serum antineutrophil cytoplasmic autoantibodies in inflammatory bowel disease are mainly associated with ulcerative colitis. A correlation study between perinuclear antineutrophil cytoplasmic autoantibodies and clinical parameters, medical, and surgical treatment. Gut 1993;34:46-50.

8 Aitola P, Miettinen A, Mattila A, et al. Effect of proctocolectomy on serum antineutrophil cytoplasmic antibodies in patients with chronic ulcerative colitis. F Clin Pathol 1995; 48:645-7.

9 Quinton JF, Sendid B, Reumaux D, et al. Anti-saccharomyces cerevisiae mannan antibodies combined with antineutrophil cerevisiae mannan antibodies combined with antineutrophil cytoplasmic autoantibodies in inflammatory bowel di
prevalence and diagnostic role. Gut 1998;42:788-91.

10 Seibold F, Brandwein S, Simpson S, et al. pANCA represents a cross-reactivity to enteric bacterial antigens. $f$ Clin Immunol 1998;18:153-60.

11 Cohavy O, Bruckner D, Gordon LK, et al. Colonic bacteria express an ulcerative colitis pANCA-related protein epitope. Infect Immun 2000;68:1542-8.

12 DeSilva HJ, deAngelis CP, Soper N, et al. Clinical and functional outcome after restorative proctocolectomy. $\mathrm{Br} \mathcal{F}$ Surg 1991;78:1039-44.

13 Salemans J, Nagengast FM, Lubbers EJ, et al. Postoperative and long-term results of ileal pouch-anal anastomosis for ulcerative colitis and familial polyposis coli. Dig Dis $S \mathrm{C}$ 1992;37:1882-9.

14 Sitzmann JV, Cartland-Burns R, et al. Rectal squamous mucosectomy and ileal anal pull-through procedures: single surgeon experience in 105 patients. Surgery 1995 ; 118:797-802.

15 Hurst RD, Molinari M, Chung TP, et al. Prospective study of the incidence, timing, and treatment of pouchitis in 104 consecutive patients after restorative proctocolectomy. Arch Surg 1996;131:497-502.

16 Madden MV, McIntyre S, Nicholls RJ. Double-blind crossover trial of metronidazole versus placebo in chronic unremitting pouchitis. Dig Dis Sci 1994;39:1193-6.

17 Aisenberg J, Wagreich J, Shim J, et al. Perinuclear anti-neutrophil cytoplasmic antibody and refractory pouchitis. Dig Dis Sci 1995;40:1866-72.

18 Yang $\mathrm{P}$, Oresland T, Jarnerot G, et al. Perinuclear antineutrophil cytoplasmic antibody in pouchitis after proctocolectomy with ileal pouch-anal anastomosis for ulcerative colitis. Scand $\mathcal{7}$ Gastroenterol 1996;31:594-8.

19 Yasuda N, Thomas P, Ellis H, et al. Perinuclear antineutrophil cytoplasmic antibodies in ulcerative colitis after restorative proctocolectomy do not correlate with the presence of pouchitis. Scand f Gastroenterol 1998;33:509-13.

20 Vecchi M, Gionchetti P, Bianchi MB, et al. p-ANCA and development of pouchitis in ulcerative colitis patients after proctocolectomy and ileoanal pouch anastomosis. Lancet 1994;344:886-7.

21 Sandborn WJ, Landers CJ, Tremaine WJ, et al. Antineutrophil cytoplasmic antibody correlates with chronic pouchitis after ileal pouch-anal anastomosis. $A m \quad \mathcal{J}$ Gastroenterol 1995;90:740-6.

22 Kaditis AG, Perrault J, Sandborn WJ, et al. Antineutrophil cytoplasmic antibody subtypes in children and adolescents after ileal pouch-anal anastomosis for ulcerative colitis. $\mathcal{F}$ Pediatr Gastroenterol Nutr 1998;26:386-92.

23 Vidrich A, Lee J, James E, et al. Segregation of pANCA antigenic recognition by DNAse treatment of neutrophils: ulcerative colitis, type 1 autoimmune hepatitis, and primary sclerosing cholangitis. F Clin Immunol 1995;15: 293-9.

24 Vasiliauskas EA, Kam LY, Karp LC, et al. Marker antibody expression stratifies Crohn's disease into immunologically homogeneous subgroups with distinct clinical characteristics. Gut 2000;47:487-96.

25 Duerr RH, Targan SR, Landers CJ, et al. Anti-neutrophil cytoplasmic antibodies in ulcerative coltis: comparison with other colitides/diarrheal illnesses. Gastroenterology 1991;100:1590-6.

26 Hardarson S, LaBrecque DR, Mitros FA, et al. Antineutrophil cytoplasmic antibody in inflammatory bowel diseases. Am f Clin Pathol 1993;99:277-81

27 Seibold F, Slametschka D, Gregor M, et al. Neutrophil autoantibodies: a genetic marker in primary cholangitis and ulcerative colitis. Gastroenterology 1994;107:532-6.

28 Sandborn WJ, Landers CJ, Tremaine WJ, et al. Association of antineutrophil cytoplasmic antibodies with resistance to treatment of left-sided ulcerative colitis: results of a pilot study. Mayo Clin Proc 1996;71:431-6.

29 Vecchi M, Bianchi MB, Sinico RA, et al. Antibodies to neutrophil cytoplasm in Italian patients with ulcerative colitis: sensitivity, specificity and recognition of putative antigens. Digestion 1994;55:34-9.

30 Vecchi M, Bianchi MB, Calabresi G, et al. Long-term observation of the perinuclear anti-cytoplasmic antibody status in ulcerative colitis patients. Scand 7 Gastroenterol 1998;33: $170-3$.

31 Boerr LA, Sambuelli AM, Katz S, et al. Clinical heterogeneity of ulcerative colitis in relation to frequency of pANCA reactivity. Gastroenterology 1995;108:A785.

32 Kock NG, Darle N, Hulten L. Ileostomy. Curr Probl Surg 1977;14:1-52

33 Fleshman JW, Cohen Z, McLeod RS, et al. The ileal reservoir and ileoanal anastomosis procedure: factors affecting technical and functional outcome. Dis Colon Rectum 1988; 31:10-16.

34 Oresland T, Fasth S, Nordgren S, et al. The clinical and functional outcome after restorative proctocolectomy: a prospective study in 100 patients. Int $\mathcal{F}$ Colorect Dis 1989;4: $50-6$

35 Kirkegaard P, Bulow S, Skov-Olsen P, et al. The first year with a J-pouch. Int $\mathcal{F}$ Colorect Dis 1990;5:148-50.

36 Svaninger G, Nordgren S, Oresland T, et al. Incidence and characteristics of pouchitis in the Kock continent ileostomy and the pelvic pouch. Scand F Gastroenterol 1993;28:695700 .

37 Stahlberg D, Gullberg K, Liljeqvist L, et al. Pouchitis following pelvic pouch operation for ulcerative colitis: incidence, cumulative risk, and risk factors. Dis Colon Rectum 1996;39:1012-18.

38 Keranen U, Luukkonen P, Jarvinen H. Functional results after restorative proctocolectomy complicated by pouchitis. Dis Colon Rectum 1997;40:764-9.

39 Patel RT, Bain I, Youngs D, et al. Cytokine production in pouchitis is similar to that in ulcerative colitis. Dis Colon Rectum 1995;38:831-7.

40 Sandborn WJ, Tremaine WJ, Batts KP, et al. Pouchitis after ileal pouch-anal anastomosis: a pouchitis disease activity index. Mayo Clin Proc 1994;69:409-15.

41 Sandborn WJ, Loftus EV, Colombel JF, et al. Utility of perinuclear anti-neutrophil cytoplasmic antibodies (pANCA), anti-Saccharomyces cerevisiae (ASCA), and antipancreatic antibodies (APA) as serologic markers in a population cohort of patients with Crohn's disease (CD: 